\title{
Real-world Evidence of Diagnostic Testing and Treatment Patterns in U.S. Breast Cancer Patients with Implications for Treatment Biomarkers from RNA- sequencing Data
}

Louis E. Fernandes ${ }^{1 *}$, Caroline G. Epstein ${ }^{1 *}$, Alexandria M. Bobe ${ }^{1 *}$, Joshua S.K. Bell ${ }^{1 *}$, Martin C. Stumpe ${ }^{1}$, Michael E. Salazar ${ }^{1}$, Ameen A. Salahudeen ${ }^{1}$, Ruth A. Pe Benito ${ }^{1}$, Calvin McCarter ${ }^{1}$, Benjamin D. Leibowitz ${ }^{1}$, Matthew Kase ${ }^{1}$, Catherine Igartua ${ }^{1}$, Robert Huether ${ }^{1}$, Ashraf Hafez ${ }^{1}$, Nike Beaubier ${ }^{1}$, Michael D. Axelson ${ }^{1}$, Mark D. Pegram², Sarah L. Sammons ${ }^{3}$, Joyce

A. O'Shaughnessy ${ }^{4}$, and Gary A. Palmer ${ }^{1}$

${ }^{*}$ co-first authors

${ }^{1}$ Tempus Labs, Chicago, IL, U.S.A.

${ }^{2}$ Stanford University School of Medicine, Stanford, CA, U.S.A.

${ }^{3}$ Department of Medicine, Duke University Medical Center, Duke University, Durham, NC, U.S.A.

${ }^{4}$ Texas Oncology and US Oncology, Dallas, TX, U.S.A.

Corresponding Author

Gary A. Palmer, gary.palmer@tempus.com

\begin{abstract}
INTRODUCTION We performed a retrospective analysis of longitudinal real-world data (RWD) from breast cancer patients to replicate results from clinical studies and demonstrate the feasibility of generating real-world evidence. We also assessed the value of transcriptome profiling as a complementary tool for determining molecular subtypes.

PATIENTS AND METHODS De-identified, longitudinal data were analyzed after abstraction from U.S. breast cancer patient records structured and stored in the Tempus database. Demographics, clinical characteristics, molecular subtype, treatment history, and survival outcomes were assessed according to strict qualitative criteria. RNA sequencing and clinical data were used to predict molecular subtypes and signaling pathway enrichment.

RESULTS The clinical abstraction cohort $(n=4,000)$ mirrored U.S. breast cancer demographics and clinical characteristics indicating feasibility for RWE generation. Among HER2+ patients, $74.2 \%$ received anti-HER 2 therapy, with $\sim 70 \%$ starting within 3 months of a positive test result. Most non-treated patients were early stage. In this RWD set, $31.7 \%$ of patients with HER2+ IHC had discordant FISH results recorded. Among patients with multiple HER2 IHC results at diagnosis, $18.6 \%$ exhibited intra-test discordance. Through development of a wholetranscriptome model to predict IHC receptor status in the molecular sequenced cohort $(n=400)$, molecular subtypes were resolved for all patients $(n=36)$ with equivocal HER2 statuses from abstracted test results. Receptor-related signaling pathways were differentially enriched between clinical molecular subtypes.
\end{abstract}

CONCLUSION RWD in the Tempus database mirrors the overall U.S. breast cancer population. These results suggest real-time, RWD analyses are feasible in a large, highly heterogeneous database. Furthermore, molecular data may aid deficiencies and discrepancies observed from breast cancer RWD. 


\section{Introduction}

A growing number of studies have explored real-world data (RWD) and

3 subsequent real-world evidence (RWE) to accelerate treatments for cancer patients.

4 RWD relates to patient information procured during routine care, while RWE is the

5 clinical evidence derived from RWD. ${ }^{1-2}$ The feasibility of this approach has increased

6 alongside technological advances and regulatory support to continuously capture and

7 integrate healthcare data sources. ${ }^{3-7}$ Several studies demonstrate the ability for RWE to

8 guide clinical development strategies, expand product labels, and address knowledge

9 gaps by examining clinical aspects not captured in clinical trials. ${ }^{8-21}$

An essential step towards strengthening RWE validity is demonstrating

11 consistency between population statistics derived from observational RWD and those

12 from controlled, experimental data. Despite the overwhelming support for RWE utility in

13 oncology, technical barriers must be addressed for RWD/RWE to reach its full clinical

14 potential. Incorporating administrative data, ancillary data, and unstructured clinical text

15 from a variety of institutions to generate RWE is a complex task. For example, no

16 standardization exists for abstracting and structuring highly heterogeneous data

17 sources, and many natural language processing algorithms cannot account for these

18 incongruencies. ${ }^{2,3}$ Consequently, clinical endpoints may not be accurately captured ${ }^{22}$

19 and even when data is properly abstracted and prepared for analysis, extraneous

20 variables in raw RWD can introduce confounding biases. ${ }^{7,23}$ Similarly, the integration of

21 omics data with RWD requires a controlled approach for large-scale data analytics. ${ }^{24}$ 
RWE and integrated omics data have the power to impact patient care. ${ }^{3,25-27}$

23 Various studies show the additive value of molecular tumor profiling with RWD for

24 clinically relevant breast cancer insights, ${ }^{8,28}$ but further advancements in the field require

25 the integration of genetic and clinical data from a variety of institutions, along with

26 omics-focused capabilities and data analytics. One potential avenue to augment the

27 value of breast cancer RWD is transcriptomics, as RNA-based gene expression

28 analyses have shown prognostic, predictive, and treatment-directing value beyond

29 DNA-sequencing insights. ${ }^{29-36}$ Whole-transcriptome RNA sequencing (RNA-seq) can

30 help classify cancer types and breast cancer biomarkers, ${ }^{37-39}$ overcoming inconclusive

31 pathology assessments, insufficient tissue quantity, and inter-observer variability of

32 immunohistochemical or in-situ hybridization assays. ${ }^{39-41}$

Here, we address some of the complexities of RWD structuring and analyses.

34 We demonstrate the feasibility of retrospective RWD analysis and test whether results

35 from clinical studies can be replicated using longitudinal RWD from a large,

36 representative breast cancer cohort. Our analyses present key clinical information, such

37 as patient demographics, clinical characteristics, molecular markers, treatment patterns,

38 and overall survival (OS) outcomes; and uncover discrepancies in real-world HER2

39 testing records. We also provide evidence supporting the integration of RWD with

40 transcriptomic profiling for clinically relevant insights through analyses of RWD and

41 molecular data from breast cancer patients sequenced by Tempus Labs. 
medRxiv preprint doi: https://doi.org/10.1101/2020.08.07.20168401; this version posted August 11, 2020. The copyright holder for this preprint (which was not certified by peer review) is the author/funder, who has granted medRxiv a license to display the preprint in perpetuity.

It is made available under a CC-BY-NC-ND 4.0 International license .

\section{Cohort Selections}

46 Two retrospective breast cancer cohorts were randomly selected from the Tempus

47 clinicogenomic database after applying clinically relevant inclusion criteria. All data were

48 de-identified in accordance with the Health Insurance Portability and Accountability Act

49 (HIPAA). Dates used for analyses were relative to the breast cancer primary diagnosis

$50(\mathrm{pdx})$ date, and year of $\mathrm{pdx}$ was randomly off-set. $\mathrm{Pdx}$ within the cohorts spanned from

51 1990-2018. The first group was a clinical abstraction (CA) cohort of 4,000 breast cancer

52 patients selected as a representative sample of RWD structured in the Tempus

53 oncology database. Records were required to have data for a pdx, pdx date, age, race,

54 sex, stage, histological subtype, and estrogen receptor (ER), progesterone receptor

55 (PR), and human epidermal growth factor receptor 2 (HER2) status. The recorded stage

56 and histological subtype were required to fall within 30 days relative to the $\mathrm{pdx}$ date,

57 while the receptor statuses may have been recorded within 30 or 50 days, depending

58 on the testing modality (see methods: Molecular Subtype Determination). A second

59 cohort was selected, the Tempus molecular sequenced (MLC) cohort, which included

60400 primary breast cancer patients with pdx dates and whose tumor biopsy underwent

61 RNA-seq and targeted DNA sequencing (DNA-seq) with the Tempus $x T(n=344), x O$

$62(n=55)$, or $x E(n=1)$ panels between 2017-2019. While only patients with reported

63 variants were included in the cohort, less than $1 \%$ of all breast cancer cases in the

64 Tempus database have no DNA variants reported.

66 IRB determined the research was exempt from IRB oversight and approved a waiver of

67 HIPAA authorization for this study. 


\section{Abstracted Molecular Markers}

70 Protein expression from immunohistochemistry $(\mathrm{IHC})$ results for ER and PR, as well as

$71 \mathrm{IHC}$ and fluorescence in-situ hybridization (FISH) results for HER2 were curated during

72 clinical data abstraction. Receptor results included abstracted equivocal, positive, or

73 negative statuses. Hormone receptor $(\mathrm{HR})$ status was classified by combinations of ER

74 and PR statuses. When available, normalized Ki67 results included indeterminant, low,

75 equivocal, moderate, or high statuses. A chi-squared test assessed the significance of

76 Ki67 test result distribution differences. Fisher's exact tests were performed for post-hoc

77 analyses, and $P$-values were adjusted for multiple hypothesis testing using Bonferroni

78 correction.

\section{Molecular Subtype Determination}

81 The molecular subtype of each CA patient was classified as HR+/HER2-, HR+/HER2+,

82 HR-/HER2+, or triple-negative breast cancer (TNBC) based on their receptor statuses at

83 diagnosis. HR statuses were determined from the most recent IHC results or physician

84 notes recorded within 30 days of the $\mathrm{pdx}$ date. $\mathrm{HR}+$ status included $\mathrm{ER}+\mathrm{PR}+, \mathrm{ER}+/ \mathrm{PR}-$

85 , and ER-/PR+. HER2 status was determined from the most recent FISH results

86 recorded within 50 days of the pdx date. In the absence of HER2 FISH data, the most

87 recent IHC result or physician note within 30 days of the pdx date was utilized.

88 References to results at "initial diagnosis" imply these 30- and 50-day time frames.

89 Molecular subtypes in the MLC cohort were determined from IHC or FISH results

90 associated with the patient pathology report. 


\section{Clinical Data Abstraction}

93 Clinical data were extracted from the Tempus real-world oncology database of

94 longitudinal structured and unstructured data from geographically diverse oncology

95 practices, including integrated delivery networks, academic institutions, and community

96 practices. Many of the records included in this study were obtained in partnership

97 through ASCO CancerLinQ. Structured data from electronic health record systems were

98 integrated with unstructured data collected from patient records via technology-enabled

99 chart abstraction and corresponding molecular data, if applicable. Data were

100 harmonized and normalized to standard terminologies from MedDRA, NCBI, NCIt,

101 NCIm, RxNorm, and SNOMED.

\section{Menopausal Status Determination}

104 Menopausal status was determined using relevant abstracted text fields when available.

105 A patient was considered premenopausal if a single, undated menopause-negative

106 (perimenopausal, premenopausal, or menstruating) status was recorded on or prior to

107 the pdx date and no menopause-positive (menopausal or postmenopausal) status was

108 indicated before diagnosis. Patients were also considered premenopausal at pdx if a

109 menopausal event was recorded after a year from the pdx date.

111 Likewise, patients with an undated menopause-positive status, and patients with a

112 menopausal or postmenopausal status recorded on or prior to the pdx date, were

113 considered postmenopausal. A patient was also considered postmenopausal if no 
114 menopausal information was available on or prior to the pdx date, but a menopausal or

115 postmenopausal status was indicated within one year after.

117 Menopausal status circumstances beyond the scope of these criteria were denoted as 118 “Unknown."

\section{Overall Survival Analysis}

121 Overall survival (OS) was calculated for all stage I-IV CA cohort patients with invasive

122 breast cancer $(\mathrm{n}=3,952)$. Patients without known relative death dates were right

123 censored at their most recent relative clinical interaction date. Survival curves were

124 generated in R using the survival (v2.43-4) and survminer (v0.4.3) packages with $P$ -

125 values calculated by log-rank tests. Results depict the percentage of surviving patients

126 per year, and are stratified based on stage and HER2, ER, and triple-negative status.

\section{Genomic Testing}

129 MLC cohort reported variants were generated from targeted DNA-seq of formalin-fixed, 130 paraffin-embedded (FFPE) slides of primary breast tumor biopsies and, when possible,

131 matched saliva or blood samples. Whole-transcriptome RNA-seq was performed on

132 samples from the same tissue block. Most samples were sequenced with the Tempus

$133 \times \mathrm{X}$ or $\mathrm{xO}$ targeted DNA-seq assays, which detect oncologic targets in solid tumors and

134 hematological malignancies as previously described. ${ }^{37,42}$ Two patient samples were

135 sequenced with an updated and refined version of the xT panel targeting clinically

136 relevant exons in 596 genes, and their reported variants were merged for analyses. 
137 Additionally, one sample in the MLC cohort was sequenced with the Tempus XE assay,

138 a whole-exome panel targeting 19,396 genes over a 39 megabase $(\mathrm{Mb})$ genomic

139 region.

140

141 Genomic Test Variant Reporting

142 Because each Tempus assay targets different gene sets, MLC cohort variant analyses

143 only included genes tested across all 400 samples. ${ }^{42,43}$ Variants were classified and

144 reported according to previously established clinical guidelines. ${ }^{37}$ Reported variants

145 were categorized as alterations, fusions, or copy number variation amplifications or

146 deletions. Alterations include variants of unknown significance (VUS), biologically

147 relevant or potentially actionable alterations, and both germline VUS and pathogenic

148 variants.

150 Tumor mutational burden (TMB)

151 TMB was calculated by dividing the number of non-synonymous mutations by the

152 adjusted panel size of the $x \mathrm{~T}$, $\mathrm{xO}$, or $\mathrm{xE}$ assay (2.4 Mb, $5.86 \mathrm{Mb}$, and $36 \mathrm{Mb}$,

153 respectively). All non-silent somatic coding mutations, including missense, indel, and

154 stop-loss variants with coverage greater than 100x and an allelic fraction greater than

$1555 \%$ were counted as non-synonymous mutations.

157 RNA-based Prediction of Molecular Subtypes

158 Transcriptome models were used to predict receptor statuses for the MLC cohort,

159 including patients lacking IHC or FISH data. Briefly, single-gene logistic models were 
medRxiv preprint doi: https://doi.org/10.1101/2020.08.07.20168401; this version posted August 11, 2020. The copyright holder for this preprint (which was not certified by peer review) is the author/funder, who has granted medRxiv a license to display the preprint in perpetuity.

It is made available under a CC-BY-NC-ND 4.0 International license .

160 trained on an independent set of Tempus RNA-sequenced breast cancer samples

161 according to the normalized gene expression of $E S R 1, P G R$, or ERBB2 using the $\mathrm{R}$ glm

162 package v2.0-16. Model performances were assessed separately for primary samples,

163 metastatic samples, and a combined set using 10-fold cross-validation (Supplemental

164 Table 2). Performance was evaluated on a testing set comprised of RNA-sequenced

165 samples in the MLC cohort with abstracted IHC or FISH results in the Tempus database

166 (ER $n=308, P R n=306$, HER2 $n=261)$. These samples were withheld from the training

167 set. Positivity thresholds for IHC prediction models were selected using Youden's J

168 statistic to optimize sensitivity and specificity.

170 Gene Expression Collection, Processing, and Normalization

171 Gene expression was generated through RNA-seq of FFPE tumor samples using an

172 exome capture-based protocol. ${ }^{37}$ Transcript-level quantification to GRCh37 was

173 performed using Kallisto 0.44. Transcript counts were then corrected for GC content

174 and length using quantile normalization and adjusted for sequencing depth via a size

175 factor method. Normalized counts in protein coding transcripts covered by the exome

176 panel were then summed to obtain gene-level counts. Subsequent expression analyses

177 were performed on $\log _{10}$-transformed counts.

179 RNA-seq Pathway Analyses

180 Gene sets were downloaded from the MSigDB website

181 (http://software.broadinstitute.org/gsea/msigdb/index.jsp), and pathway enrichment

182 scores were calculated from normalized gene expression using the ssGSEA function in 
183 Gene Set Variation Analysis (GSVA) R Bioconductor package v1.0.6. ${ }^{37,44}$ ER- and

184 HER2-related pathways were identified as those containing the terms "ESR1" or

185 "Estrogen" and "ERBB2" or "HER2," respectively. Z-scores were calculated for each set

186 of enrichment scores and the sign was reversed for any pathway containing "DN"

187 (down) or "repressed." For select analyses, the mean of the z-score across pathways

188 was calculated to produce a patient pathway metascore. With the exception of the

189 HER2 and ER signaling pathway metascore analyses, receptor status was derived from

190 both abstracted and predicted protein expression. Significance was determined by a

191 Wilcoxon test for any comparison between two groups, and a Kruskal-Wallis test for

192 comparisons between three or more groups, with $P<0.05$ considered significant. A

193 separate gene set analysis was conducted to test the difference in enrichment among

194 the four molecular subtypes relative to the 50 Hallmark pathways, a highly curated list

195 from the MSigDB database. ${ }^{45}$ To determine how patients clustered by pathway scores,

196 we performed a second UMAP analysis with enrichment scores for each Hallmark

197 pathway as features.

198

Results

Real-world evidence from a clinical abstraction breast cancer cohort

203 Patient demographics and clinical characteristics in the CA cohort

204 We first determined whether key demographic and clinical characteristics captured in

205 RWD replicate clinical studies, and found the deidentified data were consistent with 
206 previous large-scale breast cancer cohort studies (Table 1). ${ }^{46-49}$ The cohort mostly

207 comprised females $(99.3 \%, \mathrm{n}=3,970)$ with a median age at diagnosis of 61.0 years.

208 Year of diagnosis among the cohort ranged from 1990 to 2018 (Supplemental Fig. 1).

209 The self-reported race was $83.3 \%$ White $(n=3,332), 13.1 \%$ Black or African American

$210(n=523)$, and 3.6\% Asian or Pacific Islander $(n=145)$. In 2,042 females with menopausal

211 data, $87.4 \%(n=1,784)$ were postmenopausal. Abstracted stage at initial diagnosis

212 primarily consisted of stage I $(49.6 \%, n=1,986)$ and II $(33.3 \%, n=1,333)$, followed by III

$213(10.5 \%, n=420)$, IV $(5.5 \%, n=219)$, and $0(1.1 \%, 42)$. Most tumors had a histological

214 classification of invasive ductal carcinoma $(77.4 \%, n=3,095)$, and $9.5 \%(n=378)$ had an

215 invasive ductal component or were NOS. Several rare cancer types were also

216 represented.

\section{Molecular subtype determination in the CA cohort}

219 We assessed the extent to which RWD captures molecular marker information from

220 clinical testing results. The distributions of all abstracted receptor testing results at initial

221 diagnosis are shown in Fig. 1A. Consistent with previous U.S. breast cancer statistics, ${ }^{50}$

222 the most prevalent molecular subtype was HR+/HER2- $(71.5 \%, n=2,859)$, followed by

223 TNBC (12.3\%, $n=491)$ (Fig. 1B). Among HR+ patients with non-equivocal statuses,

224 most were $\mathrm{ER}+/ \mathrm{PR}+(71.0 \%, 2,839$ of 3,996$)$ followed by $\mathrm{ER}+/ \mathrm{PR}-(10.4 \%, \mathrm{n}=417)$ and

225 ER-/PR+ (1.4\%, n=57) (Fig. 1C). Lastly, abstracted Ki67 IHC test results were

226 consistent with the Ki67 expression levels typically indicative of specific breast cancer

227 subtypes (Fig. 1D). ${ }^{51-52}$ The distribution of Ki67 results differed significantly among

228 molecular subtypes (chi-squared, $P=1.75 \times 10^{-9}$ ), particularly between HR+/HER2- versus 
229 HR+/HER2+ patients $(P=0.015)$ and TNBC versus HR+/HER2- patients $\left(P=6.38 \times 10^{-9}\right)$.

230 The largest proportions of high Ki67 IHC test results were in TNBC (82.0\%, $\mathrm{n}=50$ of 61$)$

231 and HR-/HER2+ patients $(75.0 \%, \mathrm{n}=15$ of 20$)$, while most low Ki67 results were in

$232 \mathrm{HR}+/ \mathrm{HER} 2-$ patients $(44.0 \%, \mathrm{n}=140$ of 318$)$.

\section{Anti-HER2 therapy analysis in the CA cohort}

235 We next examined anti-HER2 therapy treatment patterns from longitudinal RWD.

236 Curated anti-HER2 therapies included trastuzumab, ado-trastuzumab emtansine,

237 neratinib, lapatinib and pertuzumab. Among CA cohort patients, 13.7\% $(n=546)$ were

238 HER2+ at initial diagnosis, of whom $74.2 \%(n=405)$ received anti-HER2 therapy at

239 some point in their clinical care. Approximately $70.0 \%$ of patients who received anti-

240 HER2 therapy did so within 3 months of a positive test result and the majority (73.5\%)

241 had early-stage cancer (Fig. 2A). These results are consistent with previous breast

242 cancer cohort studies. ${ }^{16,53}$ Moreover, a small portion of HER2- patients exhibited

243 evidence of receiving an anti-HER2 therapy (1.1\%, 36 of 3,352 HER2- patients) (Fig.

244 2B). Of those patients, 33.3\% $(n=12)$ had evidence of a discordant result at initial

245 diagnosis, $44.4 \%(n=16)$ had only HER2- results, and $22.2 \%(n=8)$ had a HER2-

246 equivocal or positive result recorded beyond initial diagnosis. A small portion of patients

247 ( $n=37)$ were not assigned a HER2 treatment time frame due to date quality issues.

$\underline{H E R 2 \text { test result analyses in the CA cohort }}$

250 To evaluate inter- and intra-test concordance, we compared HER2 IHC and FISH

251 results among patients with both tests conducted near initial diagnosis $(17.7 \%, n=709)$. 
252 Among patients with HER2+ IHC results and subsequent FISH testing, 62.2\% ( $\mathrm{n}=51$ of

253 82) were inter-test concordant (Supplemental Table 1), however, 31.7\% with HER2+

254 IHC were HER2- by FISH ( $\mathrm{n}=26$ of 82$)$. This discordance is larger than a previously

255 reported meta-analysis of IHC and FISH HER2 testing worldwide. ${ }^{54}$ Four of those 26

256 patients had received an anti-HER2 therapy in their clinical timeline. Among patients

257 with HER2- IHC results, 3.9\% ( $n=7$ of 182) were HER2+ by FISH, similar to historical

258 reports. ${ }^{54}$ The majority of these patients ( $n=6$ of 7 ) received anti-HER2 therapy. HER2-

259 equivocal IHC results (HER2 IHC 2+) were observed in $62.8 \%(n=445$ of 709$)$ of the

260 cohort. Among these patients with equivocal results, 7.8\% ( $n=35$ of 445) were later

261 confirmed equivocal by FISH testing. However, 80.7\% ( $n=359$ of 445$)$ had subsequent

262 HER2- and $11.5 \%(n=51$ of 445$)$ HER2+ FISH results.

263 Additionally, intra-test discordance was analyzed in patients with multiple HER2 results

264 at initial diagnosis. Among patients with multiple HER2 IHC results at diagnosis (7.1\%,

$265 \mathrm{n}=253$ of 3,561 with HER2 IHC), 18.6\% $(n=47)$ exhibited intra-test discordance. Of

266 patients with multiple HER2 FISH results $(4.5 \%, n=52$ of 1,157$), 21.2 \%(n=11)$ exhibited

267 intra-test discordance.

269 Overall survival in the CA cohort

270 OS analyses from longitudinal RWD revealed overall 5-year and 10-year survival rates

271 (92.2\% and 85.7\%, respectively) relatively consistent with average U.S. percentages

272 (Fig. 3A). ${ }^{46}$ Survival rates were expectedly high, varying by stage $(P<0.0001)$ and

273 receptor status. Stage IV patients exhibited worse OS than earlier-stage patients (Fig.

274 3B). The 5-year survival rate was $93.5 \%$ in stage I-IV HER2+ patients and $92.0 \%$ in 
medRxiv preprint doi: https://doi.org/10.1101/2020.08.07.20168401; this version posted August 11, 2020. The copyright holder for this preprint (which was not certified by peer review) is the author/funder, who has granted medRxiv a license to display the preprint in perpetuity.

It is made available under a CC-BY-NC-ND 4.0 International license .

275 HER2- patients $(P=0.45)$, with rates of $74.3 \%$ and $57.1 \%$, respectively, among stage IV

276 patients $(P=0.098)$ (Fig. 3C, 3D). The 5-year survival rate was 92.7\% among stage I-IV

277 ER+ patients and $89.8 \%$ in ER-patients $(P=0.052)$, with rates of $63.7 \%$ and $55.5 \%$,

278 respectively, among stage IV patients $(P=0.12)$ (Fig. 3E, 3F). TNBC patients had

279 significantly worse OS compared to other subtypes, with a $36.3 \% 5$-year survival rate in

280 stage IV TNBC patients compared with $65.1 \%$ among stage IV non-TNBC patients

$281 \quad(P=0.0024)$ (Fig. 3G, 3H).

282

\section{Genomic testing insights from the Tempus molecular sequenced cohort}

\section{Patient demographics and clinical characteristics in the MLC cohort}

Abstracted clinical characteristics and patient demographics from the 400 MLC cohort

290 Black or African American (14.6\%, $\mathrm{n}=35)$ and Asian or Pacific Islander patients $(5.4 \%$,

$291 \mathrm{n}=13$ ) than the CA cohort. Patients with known stage information were mostly stage II at

292 diagnosis $(38.4 \%, n=83)$, followed by stages IV $(26.4 \%, n=57)$, III $(21.3 \%, n=46)$, and I

$293(13.9 \%, n=30)$, indicating an overall higher risk population compared with the CA cohort.

294 A total of $75.0 \%(n=267)$ of tumors were invasive ductal carcinoma, with several rare 295 cancer types also represented in the cohort. 
298 The top three genes with reported alterations were TP53, PIK3CA, and GATA3, which

299 were found in $55.0 \%(n=220), 29.0 \%(n=116)$, and $13.8 \%(n=55)$ of the MLC cohort,

300 respectively (Supplemental Fig. 2A). These findings are consistent with a previous

301 analysis of The Cancer Genome Atlas breast cancer data. ${ }^{55}$ Supplemental Fig. 2B

302 shows the distribution of variant types in the 20 most frequently reported genes.

303 Assessment of patients with tumor/normal-matched DNA-seq $(n=356)$ identified 18

304 patients $(5.1 \%)$ with pathogenic germline variants in 12 NCCN-designated familial high-

305 risk genes (Supplemental Fig. 2C). This sub-population may be underrepresented as

306 exon-level duplications or deletions were not included. Among the 18 patients harboring

307 a pathogenic germline variant in any of those 12 genes, most contained variants in

308 BRCA 1 or $2(\mathrm{n}=11)$, followed by CHEK2 $(\mathrm{n}=6), \operatorname{ATM}(\mathrm{n}=3)$, and PALB2 $(\mathrm{n}=2)$. Because

309 TMB and MSI status are integrated biomarker measurements in the Tempus platform,

310 we observed a wide range of TMB across the cohort with a median of 1.7 mutations/Mb

311 (Supplemental Fig. 2D). Consistent with previous studies, ${ }^{56}$ the majority of patients

$312(84.7 \%, n=339)$ were MSI stable, while only $0.3 \%(n=1)$ were MSI high and $0.5 \%(n=2)$

313 were MSI low.

$315 \quad$ RNA-based prediction of receptor status for molecular subtypes

316 We developed a whole-transcriptome model based on 19,147 genes to predict IHC

317 receptor status and resolve molecular subtypes in the MLC cohort. Predicted RNA-

318 based subtypes largely aligned with abstracted IHC-based subtypes (Fig. 4A). Similar

319 to the literature,$^{57-58}$ transcriptome signatures differed between molecular subtypes with

320 TNBC clustering separately. Seventeen samples clustered with TNBC but were 
medRxiv preprint doi: https://doi.org/10.1101/2020.08.07.20168401; this version posted August 11, 2020. The copyright holder for this preprint (which was not certified by peer review) is the author/funder, who has granted medRxiv a license to display the preprint in perpetuity.

It is made available under a CC-BY-NC-ND 4.0 International license .

321 predicted or abstracted as another subtype, suggesting samples that cluster outside of

322 their groups may benefit from further testing or analysis. ESR1, PGR, and ERBB2 gene

323 expression correlated with their respective abstracted and predicted receptor statuses

324 (Fig. 4B).

RNA-based receptor status predictions were highly accurate for ER $(95.5 \%$,

326 AUROC 98.1\%) and HER2 (94.6\%, AUROC 93.8\%) relative to abstracted status, while

327 PR status was predicted with slightly lower accuracy (87.9\%, AUROC 95.2\%) (Fig. 5).

328 Prediction accuracy for all receptors was 92.7\%. A detailed overview of the validation

329 data and model performance are available in Supplemental Table 2. Patients with

330 incompletely abstracted molecular subtypes $(n=150)$ were classified by predicted

331 receptor statuses from the transcriptomic model. Importantly, patients with equivocal

332 HER2 statuses abstracted from IHC and/or FISH results $(n=36)$ were predicted HER2+

$333(n=7)$ or HER2- $(n=29)$ by the model.

$\underline{R N A-b a s e d ~ H E R 2 \text { and ER pathway analyses }}$

336 To further evaluate the potential for RNA-seq to enhance breast cancer clinical data, a

337 gene set enrichment analysis was conducted using the MSigDB database. First, we

338 assessed whether measuring the activity of signaling pathways may resolve ambiguous

339 or equivocal IHC and FISH test results. Multiple gene sets that putatively measure such

340 pathway activity were identified by searching for "ERBB2," "HER2," "ESR1," or

341 "Estrogen" in the MSigDB database (Supplemental Fig. 3A and 3B). Results of the

342 pathway analyses were expressed as metascores to avoid the bias introduced when

343 selecting a single pathway. HER2 IHC-positive and FISH-positive samples were 
344 enriched for HER2 activity metascores as expected, but the HER2 signaling results

345 contained substantial variability in pathway activity (Fig. 6A). Notably, the

346 GO_ERBB2_SIGNALING_PATHWAY, which directly measures HER2 activity, ${ }^{59}$

347 exhibited a robust correlation with HER2 expression ( $r=0.453)$ (Supplemental Fig. 3A)

348 and significantly different enrichments between HER2 statuses $(P=0.00031)$

349 (Supplemental Fig. 5). While ER enrichment scores were more distinct between IHC-

350 positive and IHC-negative patients, consistent with the relatively higher reliability of ER

351 IHC compared with HER2 tests, ${ }^{60-62}$ variability was also observed in the ER signaling

352 results (Fig. 6B, Supplemental Fig. 6).

353 Next, RNA-seq data were analyzed in relation to the highly curated Hallmark

354 pathway gene sets to determine the differential activation of biological pathways

355 between breast cancer subtypes. ${ }^{45}$ Most Hallmark pathways (32 of 50) exhibited

356 significantly different enrichment scores between molecular subtypes (Supplemental

357 Fig. 4A). A UMAP using only scores from these 50 pathways recapitulated the TNBC

358 clustering observed in the full-transcriptome UMAP (Fig. 6C). As expected, HR+

359 samples, but not HR- or TNBC samples, were highly enriched for two pathways related

360 to estrogen signaling (Supplemental Fig. 4B). Among HR-/HER2+ cancers, we

361 observed enrichment for pathways known to be downstream of HER2, RAS, and mTOR

362 (Fig. 6D). ${ }^{63}$ HER2-driven tumors also showed enrichment for all immune-related

363 Hallmark pathways, a finding consistent with the literature. ${ }^{64}$ Many oncogenic signaling

364 pathways were enriched in TNBC (Fig. 6E), including Wnt, mTOR, PI3K, Hedgehog,

365 and Notch, consistent with TNBC tumors' reliance on ER-, PR-, and HER2-independent

366 pathways. ${ }^{65}$ TNBC samples were also enriched for pathways related to mitotic index, as 
367 expected due to their relatively high growth rate, ${ }^{66}$ glycolysis, which is consistent with

368 their elevated Warburg effect and potentially targetable, ${ }^{67}$ and cancer/testis antigens. ${ }^{68}$

\section{Discussion}

372 The expanding utility of RWE is evident with the growing number of related studies and

373 regulatory considerations..$^{2,3,7,69}$ Compared with randomized controlled trials, however,

374 RWD analyses are complicated by a lack of standardization between records and the

375 introduction of extraneous factors, such as natural language processing errors and

376 uncontrolled confounding variables..$^{2,3,7,22-24}$ We aimed to address these concerns by 1)

377 increasing the statistical power of analyses with a relatively large cohort size, 2)

378 incorporating a variety of data sources beyond electronic health records to benefit

379 downstream analyses,,$^{1,3,22,27}$ and 3) demonstrating consistency between characteristics

380 of the real-world cohort and results from previous clinical studies.

382 clinicogenomic database, our retrospective analysis provides further evidence for the

383 feasibility and value of generating clinically relevant RWE. We first demonstrated that

384 longitudinal RWD can capture key information regarding patient clinical history,

385 treatment journey, and outcomes. Our RWD analyses generated valid RWE that

386 replicated previously published clinical results and was generally consistent with

387 established databases, indicating feasibility. Although the majority of cohort

388 characteristics were aligned with previous clinical studies, the analyses also highlighted

389 the complexities in breast cancer RWD. For instance, the proportion of pre- and post- 
390 menopausal patients was similar to previous clinical trial data, ${ }^{49}$ but menopausal status

391 was only confidently abstracted in approximately $51 \%$ of the cohort. Upon further

392 review, many RWD breast cancer studies have either applied simplified definitions of

393 menopause, such as an age cutoff, ${ }^{19}$ reported missing statuses in electronic records, ${ }^{8,70}$

394 or did not include menopausal status at all. Simplifying rules for abstraction may fill

395 these gaps in RWD, such as in defining real-world progression-free survival, but can

396 also affect the validity of conclusions. ${ }^{71,72}$

To strengthen the validity of RWE presented here, rules were established and

398 applied to perform relevant analyses and derive statistics from the cohort. For example,

399 rules described in the methods facilitated the definition of molecular subtypes from

400 multiple abstracted test results. Our HER2 test result analyses confirm the existing

401 conflict in standard testing interpretations, an issue evident by recent American Society

402 of Clinical Oncology (ASCO) guidelines, previous clinical studies, and meta-

403 analyses. ${ }^{54,73-76}$ Specifically, our findings of IHC intra-test discordance illustrate the

404 subjectivity of IHC testing, prompting standard testing improvements and biomarker

405 discovery.

407 separate cohort with complete biopsy data was selected to test the efficacy of a whole-

408 transcriptome model in predicting molecular subtypes. By combining clinical and

409 molecular data, we demonstrate transcriptome profiling is complementary to RWD and

410 can illuminate fundamental biological differences between patients. RNA-seq may

411 supplement standard testing interpretations by providing clinically relevant insights

412 when biopsy test data is inconclusive, exemplified here in the resolution of molecular 
medRxiv preprint doi: https://doi.org/10.1101/2020.08.07.20168401; this version posted August 11, 2020. The copyright holder for this preprint (which was not certified by peer review) is the author/funder, who has granted medRxiv a license to display the preprint in perpetuity.

It is made available under a CC-BY-NC-ND 4.0 International license .

413 subtypes for patients with equivocal statuses. Addressing these cases is critical in the

414 evolving treatment landscape, as molecular subtypes are key criteria for breast cancer

415 treatment decisions.

$416 \quad$ Furthermore, our signaling pathway investigation uncovered potential pathway-

417 related therapeutic targets, such as oncogenic signaling via the mTOR pathway, for

418 subtypes like TNBC with limited pharmacotherapies available. RNA pathway analyses

419 can also elucidate treatment-related tumor characteristics not captured by standard

420 diagnostic and prognostic tests, such as additional biomarkers or amplifications that

421 may be targetable in HER2+ breast cancer patients. ${ }^{77-79}$ Expression-based immune

422 signatures can also predict response to neoadjuvant treatment with several

423 experimental agents/combinations added to standard chemotherapy, including the

424 addition of pembrolizumab in early-stage TNBC. ${ }^{80}$ Biomarker selection of

425 immunotherapy in early-stage TNBC will become imperative to therapeutic strategies

426 given its substantial toxicity.

\section{Conclusion}

429 The Tempus data pipeline integrates longitudinal RWD and comprehensive

430 molecular sequencing data into a structured clinicogenomic database capable of

431 generating valid clinical evidence in real-time. While RWD are inherently complex,

432 cancer cohort selection and data insights are feasible using structured data sources and

433 strictly defined analysis criteria. Finally, integrating RNA-seq data with RWD can

434 improve clinically actionable evidence related to clinical markers, potential therapeutic

435 targets, and optimal therapy selection in breast cancer. 


\section{Clinical Practice Points}

439 - The feasibility of real-world data (RWD) analysis has increased alongside technological advances and regulatory support to continuously capture and integrate healthcare data sources. Several studies demonstrate the ability for real-world evidence (RWE) to guide clinical development strategies, expand product labels, and address knowledge gaps by examining clinical aspects not captured in clinical trials. heterogenous structured and unstructured sources is often challenged by various technical barriers. Lack of standardization between electronic records, underpowered natural language processing tools, and uncontrolled extraneous variables threaten the validity of well-sourced RWE.

450 - Our RWD analyses followed strict qualitative criteria to produce RWE of demographics, clinical characteristics, molecular subtype, treatment history, and survival outcomes from a large, heterogeneous database. Importantly, the results were mostly consistent with data from previous clinical studies, suggesting feasibility of generating valid RWE. We also demonstrate the value of integrating omics data with RWD through the use of whole-transcriptome analyses in relevant breast cancer signaling pathways and a predictive model for receptor statuses. 
- These data provide rational for use of the Tempus clinicogenomic database to generate RWE and conduct real-time, hypothesis-driven analyses of large RWD cohorts in the future. Clinicians may utilize these large-scale databases to circumvent the restrictive exclusion criteria of controlled studies, clarify real-world patient needs, and aid the development of clinical trials. Furthermore, our results suggest molecular data may bolster deficiencies in standard breast cancer diagnostic tests.

\section{Acknowledgments}

467 We are thankful to ASCO CancerLinQ for their partnership and the clinical data, 468 operations, data science, engineering, pathology, and lab teams at Tempus Labs. We

469 sincerely thank the entire clinical data abstraction team, Jeff Ottens, and April Manhertz.

470 We thank Kelly McKinnon for proofreading and figure assembly and design. We thank

471 Kevin White and Kimberly Blackwell for scientific review and discussion of the

472 manuscript. We thank Hailey Lefkofsky for initial discussions and Eric Lefkofsky for his

473 support and discussions.

474

475

476

477

478

479 
medRxiv preprint doi: https://doi.org/10.1101/2020.08.07.20168401; this version posted August 11, 2020. The copyright holder for this preprint (which was not certified by peer review) is the author/funder, who has granted medRxiv a license to display the preprint in perpetuity.

It is made available under a CC-BY-NC-ND 4.0 International license .

\section{References}

1. Nabhan C, Klink A, Prasad V: Real-world Evidence-What Does It Really Mean? JAMA Oncology 5:781, 2019

2. Administration USFD: Framework for the FDA's Real-world Evidence Program. 2018

3. Jourquin J, Reffey SB, Jernigan C, et al: Susan G. Komen Big Data for Breast Cancer Initiative: How Patient Advocacy Organizations Can Facilitate Using Big Data to Improve Patient Outcomes. JCO Precision Oncology:1-9, 2019

4. Administration USFD: Submitting Documents Using Real-World Data and RealWorld Evidence to FDA for Drugs and Biologics Guidance for Industry, 2019

5. Administration USFD: Use of Electronic Health Record Data in Clinical Investigations: Guidance for Industry. 2018

6. Administration USFD: Use of Real-World Evidence to Support Regulatory Decision-Making for Medical Devices: Guidance for Industry and Food and Drug Administration Staff, 2017

7. Khozin S, Blumenthal GM, Pazdur R: Real-world Data for Clinical Evidence Generation in Oncology. JNCl: Journal of the National Cancer Institute 109, 2017

8. Quek RGW, Mardekian J: Clinical Outcomes, Treatment Patterns, and Health Resource Utilization Among Metastatic Breast Cancer Patients with Germline BRCA1/2 Mutation: A Real-World Retrospective Study. Advances in Therapy 36:708-720, 2019

9. Taylor-Stokes G, Mitra D, Waller J, et al: Treatment patterns and clinical outcomes among patients receiving palbociclib in combination with an aromatase inhibitor or fulvestrant for HR+/HER2-negative advanced/metastatic breast cancer in real-world settings in the US: Results from the IRIS study. The Breast 43:22-27, 2019

10. Khozin S, Carson KR, Zhi J, et al: Real-World Outcomes of Patients with Metastatic Non-Small Cell Lung Cancer Treated with Programmed Cell Death Protein 1 Inhibitors in the Year Following U.S. Regulatory Approval. The Oncologist 24:648-656, 2019

11. Martina R, Jenkins D, Bujkiewicz $S$, et al: The inclusion of real world evidence in clinical development planning. Trials 19, 2018

12. Harrell M, Fabbri D, Levy M: Analysis of Adjuvant Endocrine Therapy in Practice From Electronic Health Record Data of Patients With Breast Cancer. JCO Clinical Cancer Informatics:1-8, 2017

13. El-Galaly TC, Jakobsen $\mathrm{LH}$, Hutchings $M$, et al: Routine Imaging for Diffuse Large B-Cell Lymphoma in First Complete Remission Does Not Improve Post-Treatment Survival: A Danish-Swedish Population-Based Study. Journal of Clinical Oncology 33:3993-3998, 2015

14. Przepiorka D, Ko CW, Deisseroth A, et al: FDA Approval: Blinatumomab. Clinical Cancer Research 21:4035-4039, 2015

15. Hernandez AF, Fleurence RL, Rothman RL: The ADAPTABLE Trial and PCORnet: Shining Light on a New Research Paradigm. Annals of Internal Medicine 163:635-636, 2015 
medRxiv preprint doi: https://doi.org/10.1101/2020.08.07.20168401; this version posted August 11, 2020. The copyright holder for this preprint (which was not certified by peer review) is the author/funder, who has granted medRxiv a license to display the preprint in perpetuity.

16. Dawood S, Broglio K, Buzdar AU, et al: Prognosis of Women With Metastatic Breast Cancer byHER2Status and Trastuzumab Treatment: An Institutional-Based Review. Journal of Clinical Oncology 28:92-98, 2010

17. Pfizer: U.S. FDA APPROVES IBRANCE ${ }^{\circledR}$ (PALBOCICLIB) FOR THE TREATMENT OF MEN WITH HR+, HER2- METASTATIC BREAST CANCER, 2019

18. Administration USFD: $<$ IBRANCE ${ }^{\circledR}$ (palbociclib) capsules, for oral use - Label.pdf $>$.

19. Gierach GL, Curtis RE, Pfeiffer RM, et al: Association of Adjuvant Tamoxifen and Aromatase Inhibitor Therapy With Contralateral Breast Cancer Risk Among US Women With Breast Cancer in a General Community Setting. JAMA Oncology 3:186, 2017

20. Daniels B, Kiely BE, Lord SJ, et al: Long-term survival in trastuzumab-treated patients with HER2-positive metastatic breast cancer: real-world outcomes and treatment patterns in a whole-of-population Australian cohort (2001-2016). Breast Cancer Research and Treatment 171:151-159, 2018

21. McNamara DM, Goldberg SL, Latts L, et al: Differential impact of cognitive computing augmented by real world evidence on novice and expert oncologists. Cancer Medicine, 2019

22. Cowie MR, Blomster JI, Curtis LH, et al: Electronic health records to facilitate clinical research. Clinical Research in Cardiology 106:1-9, 2017

23. Skovlund E, Leufkens HGM, Smyth JF: The use of real-world data in cancer drug development. European Journal of Cancer 101:69-76, 2018

24. Warner JL, Jain SK, Levy MA: Integrating cancer genomic data into electronic health records. Genome Medicine 8:113, 2016

25. Shimelis H, Laduca H, Hu C, et al: Triple-Negative Breast Cancer Risk Genes Identified by Multigene Hereditary Cancer Panel Testing. JNCl: Journal of the National Cancer Institute 110:855-862, 2018

26. Vallon-Christersson J, Häkkinen J, Hegardt C, et al: Cross comparison and prognostic assessment of breast cancer multigene signatures in a large population-based contemporary clinical series. Scientific Reports 9, 2019

27. Győrffy B, Pongor L, Bottai G, et al: An integrative bioinformatics approach reveals coding and non-coding gene variants associated with gene expression profiles and outcome in breast cancer molecular subtypes. British Journal of Cancer 118:1107-1114, 2018

28. Avazpour N, Hajjari M, Tahmasebi Birgani M: HOTAIR: A Promising Long Noncoding RNA with Potential Role in Breast Invasive Carcinoma. Frontiers in Genetics 8, 2017

29. Plitas G, Konopacki C, Wu K, et al: Regulatory T Cells Exhibit Distinct Features in Human Breast Cancer. Immunity 45:1122-1134, 2016

30. Guo W, Wang Q, Zhan Y, et al: Transcriptome sequencing uncovers a three-long noncoding RNA signature in predicting breast cancer survival. Scientific Reports 6:27931, 2016

31. Craig DW, O'Shaughnessy JA, Kiefer JA, et al: Genome and Transcriptome Sequencing in Prospective Metastatic Triple-Negative Breast Cancer Uncovers Therapeutic Vulnerabilities. Molecular Cancer Therapeutics 12:104-116, 2013

32. Zoon CK, Starker EQ, Wilson AM, et al: Current molecular diagnostics of breast 564 9:455-466, 2009 
medRxiv preprint doi: https://doi.org/10.1101/2020.08.07.20168401; this version posted August 11, 2020. The copyright holder for this preprint (which was not certified by peer review) is the author/funder, who has granted medRxiv a license to display the preprint in perpetuity. It is made available under a CC-BY-NC-ND 4.0 International license .

607

608

33. BreastCancer.org: MammaPrint Test, 2019

34. Beaubier $\mathrm{N}$, Bontrager $\mathrm{M}$, Huether $\mathrm{R}$, et al: Integrated genomic profiling expands clinical options for patients with cancer. Nature Biotechnology, 2019

35. Michuda J, Igartua C, Taxter T, et al: Transcriptome-based cancer type prediction for tumors of unknown origin. Journal of Clinical Oncology 37:3081-3081, 2019

36. Grewal JK, Tessier-Cloutier B, Jones M, et al: Application of a Neural Network Whole Transcriptome-Based Pan-Cancer Method for Diagnosis of Primary and Metastatic Cancers. JAMA Network Open 2:e192597, 2019

37. Brueffer C, Vallon-Christersson J, Grabau D, et al: Clinical Value of RNA Sequencing-Based Classifiers for Prediction of the Five Conventional Breast Cancer Biomarkers: A Report From the Population-Based Multicenter Sweden Cancerome Analysis Network-Breast Initiative. JCO Precision Oncology:1-18, 2018

38. Fumagalli D, Blanchet-Cohen A, Brown D, et al: Transfer of clinically relevant gene expression signatures in breast cancer: from Affymetrix microarray to Illumina RNASequencing technology. 15:1008, 2014

39. Gill J, Prasad V: Improving observational studies in the era of big data. The Lancet 392:716-717, 2018

40. Miksad RA, Abernethy AP: Harnessing the Power of Real-World Evidence (RWE): A Checklist to Ensure Regulatory-Grade Data Quality. Clinical Pharmacology \& Therapeutics 103:202-205, 2018

41. Gyawali B, Parsad S, Feinberg BA, et al: Real-World Evidence and Randomized Studies in the Precision Oncology Era: The Right Balance. JCO Precision Oncology:1-5, 2017

42. Beaubier $N$, Tell $R$, Huether $R$, et al: Clinical validation of the Tempus $\times O$ assay. Oncotarget 9, 2018

43. Beaubier N, Tell R, Lau D, et al: Clinical validation of the tempus xT nextgeneration targeted oncology sequencing assay. Oncotarget 10, 2019

44. Hänzelmann S, Castelo R, Guinney J: GSVA: gene set variation analysis for microarray and RNA-Seq data. BMC Bioinformatics 14:7, 2013

45. Liberzon A, Birger C, Thorvaldsdóttir $\mathrm{H}$, et al: The Molecular Signatures Database Hallmark Gene Set Collection. Cell Systems 1:417-425, 2015

46. Society AC: Breast Cancer Facts \& Figures 2019-2020, in Society AC (ed), 2019

47. Institute NC: SEER Cancer Statistics Review 1975-2016, 2019

48. Iqbal J, Ginsburg O, Rochon PA, et al: Differences in Breast Cancer Stage at Diagnosis and Cancer-Specific Survival by Race and Ethnicity in the United States. JAMA 313:165, 2015

49. Goss PE, Ingle JN, Martino S, et al: Impact of premenopausal status at breast cancer diagnosis in women entered on the placebo-controlled NCIC CTG MA17 trial of extended adjuvant letrozole. Annals of Oncology 24:355-361, 2013

50. Desantis CE, Ma J, Gaudet MM, et al: Breast cancer statistics, 2019. CA: A Cancer Journal for Clinicians, 2019

51. Arena V, Pennacchia I, Vecchio FM, et al: ER-/PR+/HER2- breast cancer type shows the highest proliferative activity among all other combined phenotypes and is more common in young patients: Experience with 6643 breast cancer cases. The Breast Journal 25:381-385, 2019 
medRxiv preprint doi: https://doi.org/10.1101/2020.08.07.20168401; this version posted August 11, 2020. The copyright holder for this preprint (which was not certified by peer review) is the author/funder, who has granted medRxiv a license to display the preprint in perpetuity. It is made available under a CC-BY-NC-ND 4.0 International license .

52. Nahed AS, Shaimaa MY: Ki-67 as a prognostic marker according to breast cancer molecular subtype. Cancer Biology \& Medicine 13:496, 2016

53. Gullo $G$, Walsh $N$, Fennelly $D$, et al: Impact of timing of trastuzumab initiation on long-term outcome of patients with early-stage HER2-positive breast cancer: the "one thousand HER2 patients" project. British Journal of Cancer 119:374-380, 2018

54. Bahreini F, Soltanian AR, Mehdipour P: A meta-analysis on concordance between immunohistochemistry (IHC) and fluorescence in situ hybridization (FISH) to detect HER2 gene overexpression in breast cancer. Breast Cancer 22:615-25, 2015

55. Network TCGA: Comprehensive molecular portraits of human breast tumours. Nature 490:61-70, 2012

56. Cortes-Ciriano I, Lee S, Park W-Y, et al: A molecular portrait of microsatellite instability across multiple cancers. Nature Communications 8:15180, 2017

57. Bradford JR, Cox A, Bernard P, et al: Consensus Analysis of Whole Transcriptome Profiles from Two Breast Cancer Patient Cohorts Reveals Long Non-Coding RNAs Associated with Intrinsic Subtype and the Tumour Microenvironment. PLOS ONE 11:e0163238, 2016

58. Eswaran J, Cyanam D, Mudvari P, et al: Transcriptomic landscape of breast cancers through mRNA sequencing. Scientific Reports 2, 2012

59. Smid M, Wang Y, Zhang Y, et al: Subtypes of Breast Cancer Show Preferential Site of Relapse. Cancer Research 68:3108-3114, 2008

60. Allott EH, Geradts J, Sun X, et al: Intratumoral heterogeneity as a source of discordance in breast cancer biomarker classification. Breast Cancer Research 18, 2016

61. Robertson S, Rönnlund C, De Boniface J, et al: Re-testing of predictive biomarkers on surgical breast cancer specimens is clinically relevant. Breast Cancer Research and Treatment 174:795-805, 2019

62. Dekker TJA, Smit VTHBM, Hooijer GKJ, et al: Reliability of core needle biopsy for determining ER and HER2 status in breast cancer. 24:931-937, 2013

63. Hare $\mathrm{SH}$, Harvey AJ: mTOR function and therapeutic targeting in breast cancer. American journal of cancer research 7:383-404, 2017

64. Holgado E, Perez-Garcia J, Gion $M$, et al: Is there a role for immunotherapy in HER2-positive breast cancer? npj Breast Cancer 4, 2018

65. Wu N, Zhang J, Zhao J, et al: Precision medicine based on tumorigenic signaling pathways for triple-negative breast cancer. Oncology letters 16:4984-4996, 2018

66. Elsawaf Z, Sinn H-P: Triple-Negative Breast Cancer: Clinical and Histological Correlations. Breast Care 6:273-278, 2011

67. O'Neill S, Porter RK, McNamee N, et al: 2-Deoxy-D-Glucose inhibits aggressive triple-negative breast cancer cells by targeting glycolysis and the cancer stem cell phenotype. Scientific Reports 9, 2019

68. Thomas R, Al-Khadairi G, Roelands J, et al: NY-ESO-1 Based Immunotherapy of Cancer: Current Perspectives. Frontiers in Immunology 9, 2018

69. Research FoC: Blueprint for Breakthrough: Exploring the Utility of Real World Evidence (RWE), 2016

70. Pobiruchin M, Bochum S, Martens UM, et al: A method for using real world data in breast cancer modeling. Journal of Biomedical Informatics 60:385-394, 2016 
medRxiv preprint doi: https://doi.org/10.1101/2020.08.07.20168401; this version posted August 11, 2020. The copyright holder for this preprint (which was not certified by peer review) is the author/funder, who has granted medRxiv a license to display the preprint in perpetuity.

It is made available under a CC-BY-NC-ND 4.0 International license .

71. Zare S, Rong J, Daehne S, et al: Implementation of the 2018 American Society of Clinical Oncology/College of American Pathologists Guidelines on HER2/neu Assessment by FISH in breast cancers: predicted impact in a single institutional cohort. Modern Pathology 32:15661573,2019

72. Wolff AC, Hammond MEH, Allison KH, et al: Human Epidermal Growth Factor Receptor 2 Testing in Breast Cancer: American Society of Clinical Oncology/College of American Pathologists Clinical Practice Guideline Focused Update. Journal of Clinical Oncology 36:21052122, 2018

73. von Minckwitz G, Procter M, de Azambuja E, et al: Adjuvant Pertuzumab and Trastuzumab in Early HER2-Positive Breast Cancer. New England Journal of Medicine 377:122131, 2017

74. Fehrenbacher L, Cecchini R, Geyer C, et al: Abstract GS1-02: NSABP B-47 (NRG oncology): Phase III randomized trial comparing adjuvant chemotherapy with adriamycin (A) and cyclophosphamide (C) $\rightarrow$ weekly paclitaxel (WP), or docetaxel (T) and C with or without a year of trastuzumab $(\mathrm{H})$ in women with node-positive or high-risk node-negative invasive breast cancer (IBC) expressing HER2 staining intensity of IHC 1+ or 2+ with negative FISH (HER2-Low IBC). Cancer Research 78:GS1-02-GS1-02, 2018

75. Loi S, Giobbie-Hurder A, Gombos A, et al: Pembrolizumab plus trastuzumab in trastuzumab-resistant, advanced, HER2-positive breast cancer (PANACEA): a single-arm, multicentre, phase 1b-2 trial. The Lancet Oncology 20:371-382, 2019

76. Ayoub NM, Al-Shami KM, Yaghan RJ: Immunotherapy for HER2-positive breast cancer: recent advances and combination therapeutic approaches. Breast Cancer: Targets and Therapy Volume 11:53-69, 2019

77. Sharma A, Koldovsky U, Xu S, et al: HER-2 pulsed dendritic cell vaccine can eliminate HER-2 expression and impact ductal carcinoma in situ. 118:4354-4362, 2012

\section{Figure and Table Legends}

Table 1. Demographics and clinical characteristics of the clinical abstraction and Tempus molecular sequenced cohort.

Figure 1. Breast cancer molecular biomarkers and subtypes in the clinical abstraction cohort. (A) The number of patients with positive, negative, or equivocal IHC or FISH test results for ER, PR, HR, and HER2 status at initial diagnosis. (B) The distributions of breast cancer molecular subtypes as determined by abstracted ER, PR, and HER2 test 
688 results at initial diagnosis, and (C) the distribution of ER and PR status combinations

689 across the cohort. (D) The number of patients with high, moderate, low, indeterminate,

690 or equivocal Ki67 IHC test results or status-indicating physician notes at initial

691 diagnosis, separated by molecular subtype.

692

693 Figure 2. Anti-HER2 treatment by HER2 status in the clinical abstraction cohort. Anti-

694 HER2 treatment initiation patterns among (A) HER2+ and (B) HER2- patients who

695 received anti-HER2 therapy at some point in their clinical care. M, month; $Y$, year.

697 Figure 3. Overall survival from primary diagnosis dates in the clinical abstraction cohort.

698 Ten-year survival probability in (A) all patients and (B) stage I-IV patients stratified by 699 stage. Five-year survival probabilities stratified by HER2 status in (C) all patients and 700 (D) stage IV patients, ER status in (E) all patients and $(F)$ stage IV patients, and TNBC 701 status in $(\mathrm{G})$ all patients and $(\mathrm{H})$ stage IV patients.

703 Figure 4. RNA-based receptor status prediction analysis of the Tempus molecular 704 sequenced cohort. (A) UMAP transcriptome clustering of 19,147 genes in the cohort 705 color-coded by molecular subtype. Circles correspond to samples with available IHC or 706 FISH test results for all proteins and $\mathrm{X}$ symbols correspond to patients with predicted 707 status for at least one protein. (B) Relationship between ER, PR, and HER2 receptor 708 status and $\log _{10}$-transformed, normalized gene expression of ESR1, PGR, and ERBB2.

709 Left panels represent samples with available receptor status from abstracted test 
medRxiv preprint doi: https://doi.org/10.1101/2020.08.07.20168401; this version posted August 11, 2020. The copyright holder for this preprint (which was not certified by peer review) is the author/funder, who has granted medRxiv a license to display the preprint in perpetuity.

It is made available under a CC-BY-NC-ND 4.0 International license .

710 results, while right panels represent transcriptome-based receptor status predictions.

711 HER2 predictions for samples reported as equivocal are plotted as white dots.

713 Figure 5. Single-gene logistic model performance for ER, PR, and HER2 status

714 prediction in the Tempus molecular sequenced cohort. The (A) specificity and

715 sensitivity, and (B) precision and recall of transcriptome-based receptor status

716 predictions were evaluated on a testing set comprised of cohort RNA-sequenced

717 samples with abstracted receptor status results in the Tempus database. (C) Confusion

718 matrices depicting transcriptome-based ER, PR, and HER2 status prediction

719 performance.

720

721 Figure 6: RNA-seq breast cancer pathway analyses of the Tempus molecular

722 sequenced cohort. (A) HER2 and (B) ER pathway metascores for patients with

723 abstracted HER2 IHC or FISH test results. (C) UMAP of 50 Hallmark enrichment

724 scores. Patients with molecular subtypes based on at least one abstracted receptor

725 status are depicted by circles, while patients with molecular subtypes determined

726 exclusively from RNA-predicted statuses are depicted by $\mathrm{X}$ symbols. Distribution of

727 enrichment Z-scores for (D) HR-/HER2+ and (E) TNBC relevant pathways.

728

729 Supplemental Figure and Table Legends

730

731 Supplemental Figure 1. Patients grouped by year of initial diagnosis. The distribution

732 of patients by year of initial diagnosis across the clinical abstraction cohort. 
734 Supplemental Figure 2. Molecular characteristics of the Tempus molecular sequenced

735 cohort. (A) The distribution of patients with variants in the most frequently reported

736 genes across the cohort. The number of patients harboring mutations in each gene are

737 shown above the bars. (B) The number of variants classified as alterations,

738 amplifications, or deletions within each of the most frequently reported genes in the

739 cohort. (C) The distribution of patients with pathogenic germline alterations in NCCN-

740 designated familial high-risk genes and (D) TMB across the cohort.

742 Supplemental Figure 3. Breast cancer pathway analyses from RNA-seq data of the

743 Tempus molecular sequenced cohort according to MSigDB and Hallmark pathways. (A)

744 Pearson correlation between ERBB2 expression and enrichment scores (GSVA) for

745 each HER2-related pathway in MSigDB among the cohort. (B) Correlation between

746 ESR1 expression and enrichment scores for each ER-related pathway in MSigDB

747 among the cohort.

749 Supplemental Figure 4. (A) For each Hallmark pathway, the significance of differential

750 enrichment between molecular subtypes was determined by a Kruskal-Wallis test of the

751 enrichment scores. The vertical line indicates $P=0.001$ and any value to the right of the

752 line was considered significant. (B) Distributions of z-scores among HR+/HER2- (blue),

$753 \mathrm{HR}+/ \mathrm{HER} 2+$ (green), HR-/HER2+ (orange), and TNBC (grey) patients for the two

754 estrogen response Hallmark pathways with the most significant differential enrichments

755 between molecular subtypes. 
757 Supplemental Figure 5. Distribution of enrichment z-scores for each HER2-related

758 pathway in MSigDB among patients in the Tempus molecular sequenced cohort.

759 Patients with negative (blue), equivocal (orange), or positive (green) abstracted or

760 predicted HER2 test results are shown. The $P$-values listed for each pathway represent

761 the results of a Kruskal-Wallis test for the difference between enrichment scores from

762 HER2-, HER2-equivocal, and HER2+ patients.

764 Supplemental Figure 6. Distribution of enrichment z-scores for each ER-related

765 pathway in MSigDB among patients in the Tempus molecular sequenced cohort.

766 Patients with negative (blue) or positive (green) abstracted or predicted ER test results

767 are shown. The $P$-values listed for each pathway represent the results of a Wilcox rank

768 sum test for the difference between enrichment z-scores from ER+ and ER-patients.

770 Supplemental Table 1. Inter-test comparison of HER2 status from IHC and FISH

771 results among patients in the clinical abstraction cohort with both tests conducted at

772 initial diagnosis.

773

774 Supplemental Table 2. Single-gene logistic model performance results for RNA-based

775 predictions of ER, PR, and HER2 status in the Tempus molecular sequenced cohort. 
A

HER2+ Patients Receiving Anti-HER2 Therapy

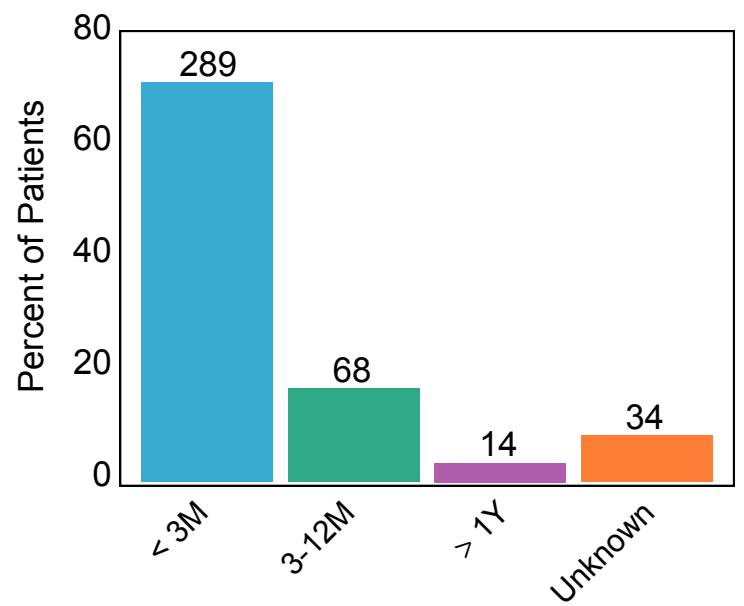

Time from Diagnosis
B

HER2- Patients Receiving Anti-HER2 Therapy

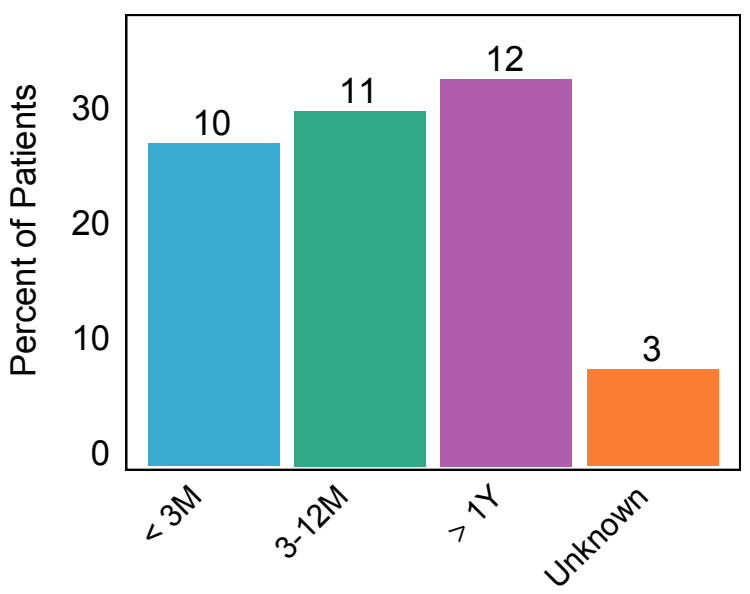

Time from Diagnosis 
Figure 3

\section{Overall survival from date of primary diagnosis}

$A$
$\frac{7}{\overline{0}}$
$\frac{0}{\pi}$
$\frac{0}{0}$
$\frac{2}{2}$
$\stackrel{2}{\vdots}$
$\omega$

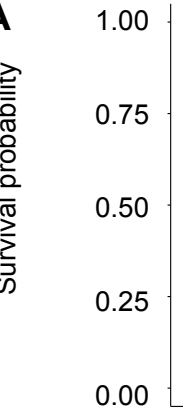

$\begin{array}{rcrrrrr}\text { Years } & 0 & 2 & 4 & 6 & 8 & 10 \\ \text { All }(n) & 3952 & 2675 & 1866 & 1199 & 785 & 473\end{array}$

C

C
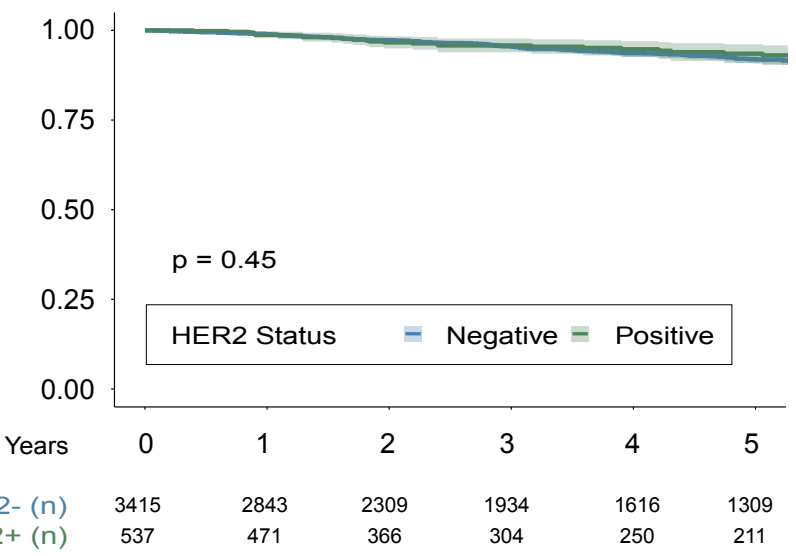

$E$
7
0
0
0
0
0
0
0
0

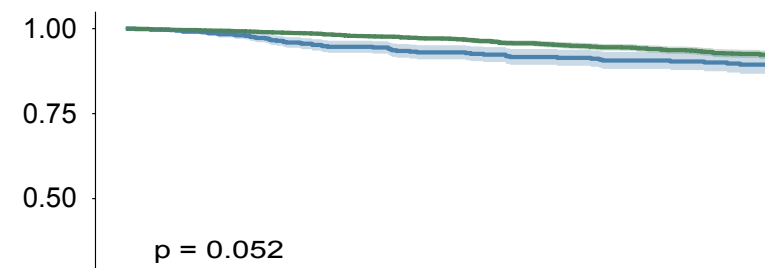

$\mathbf{G}$

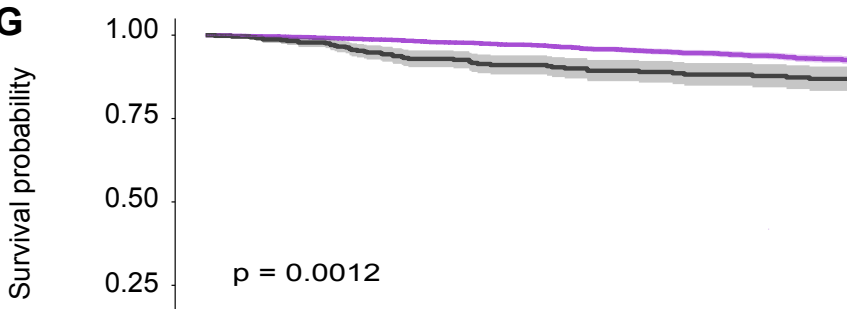

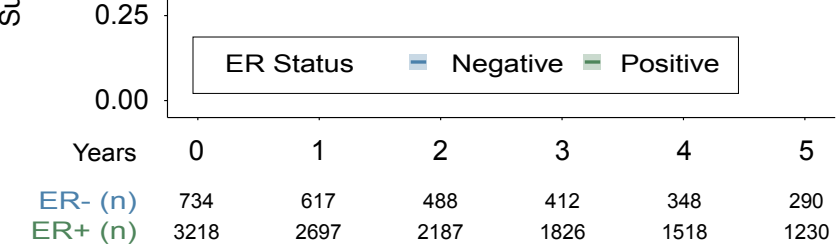

B

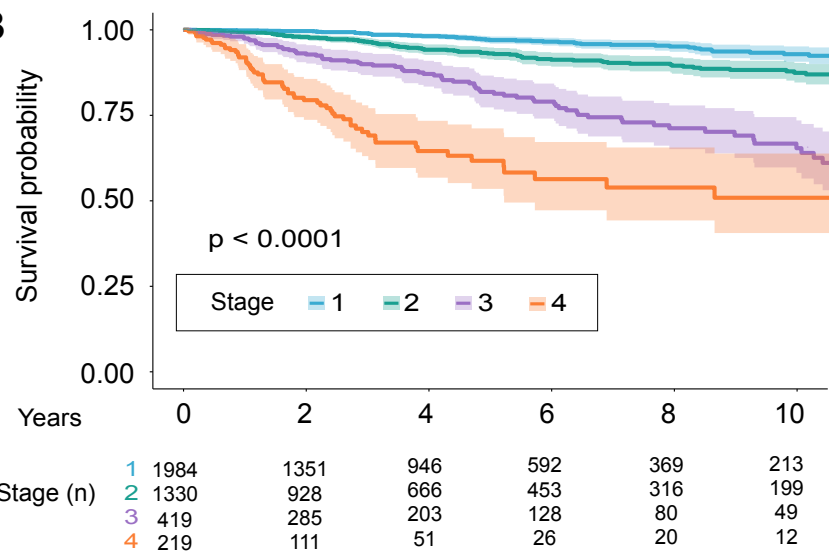

D

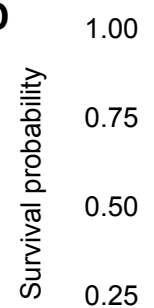

0.25

$p=0.098$

Stage 4 HER2 Status = Negative $=$ Positive $\begin{array}{lllllll}\text { Years } & 0 & 1 & 2 & 3 & 4 & 5\end{array}$

$\begin{array}{lllllll}\text { HER2- }(\mathrm{n}) & 174 & 131 & 82 & 48 & 36 & 26\end{array}$ $\begin{array}{lllllll}\mathrm{HER} 2+(\mathrm{n}) & 45 & 37 & 29 & 21 & 15 & 11\end{array}$

$\mathbf{F}$

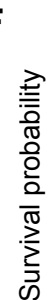

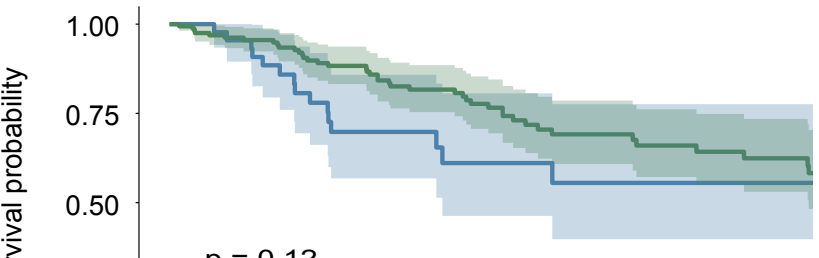

$\mathrm{p}=0.12$

0.25

Stage 4 ER Status $=$ Negative $=$ Positive

0.00

Years

ER- (n)

$E R+(n)$

H

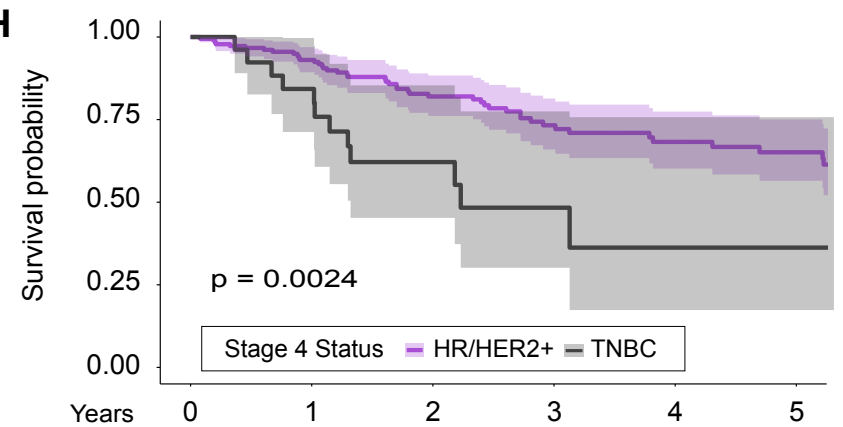


Figure 4

A

UMAP Transcriptome Clustering

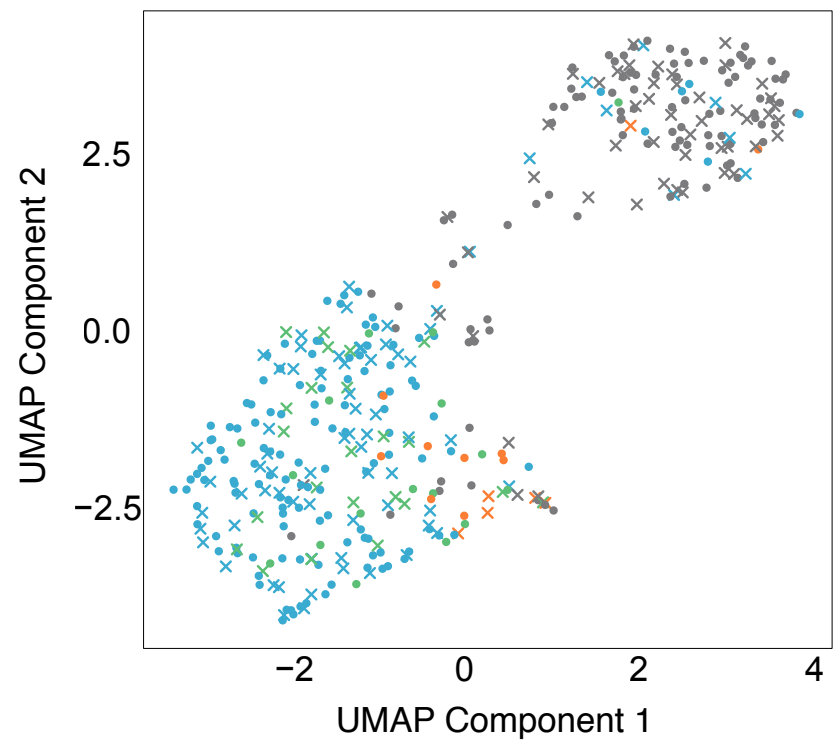

- Abstracted OHR+/HER2- OHR-/HER2+ $\times$ Predicted $\bigcirc \mathrm{HR}+/ \mathrm{HER} 2+\quad \mathrm{TNBC}$
B Correlation Between Gene and Protein Expression

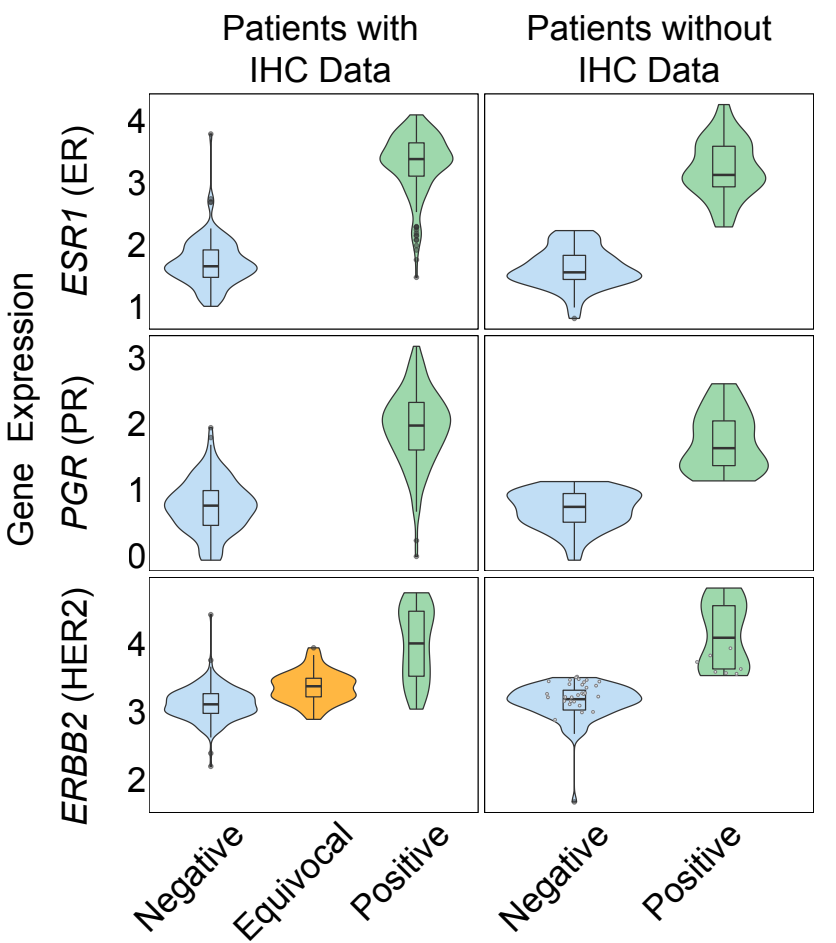


A

B

Specificity and Sensitivity of IHC Status Predictions

Precision and Recall of IHC Status Predictions

AUCs for ER: .98, PR: .95, HER2: .94
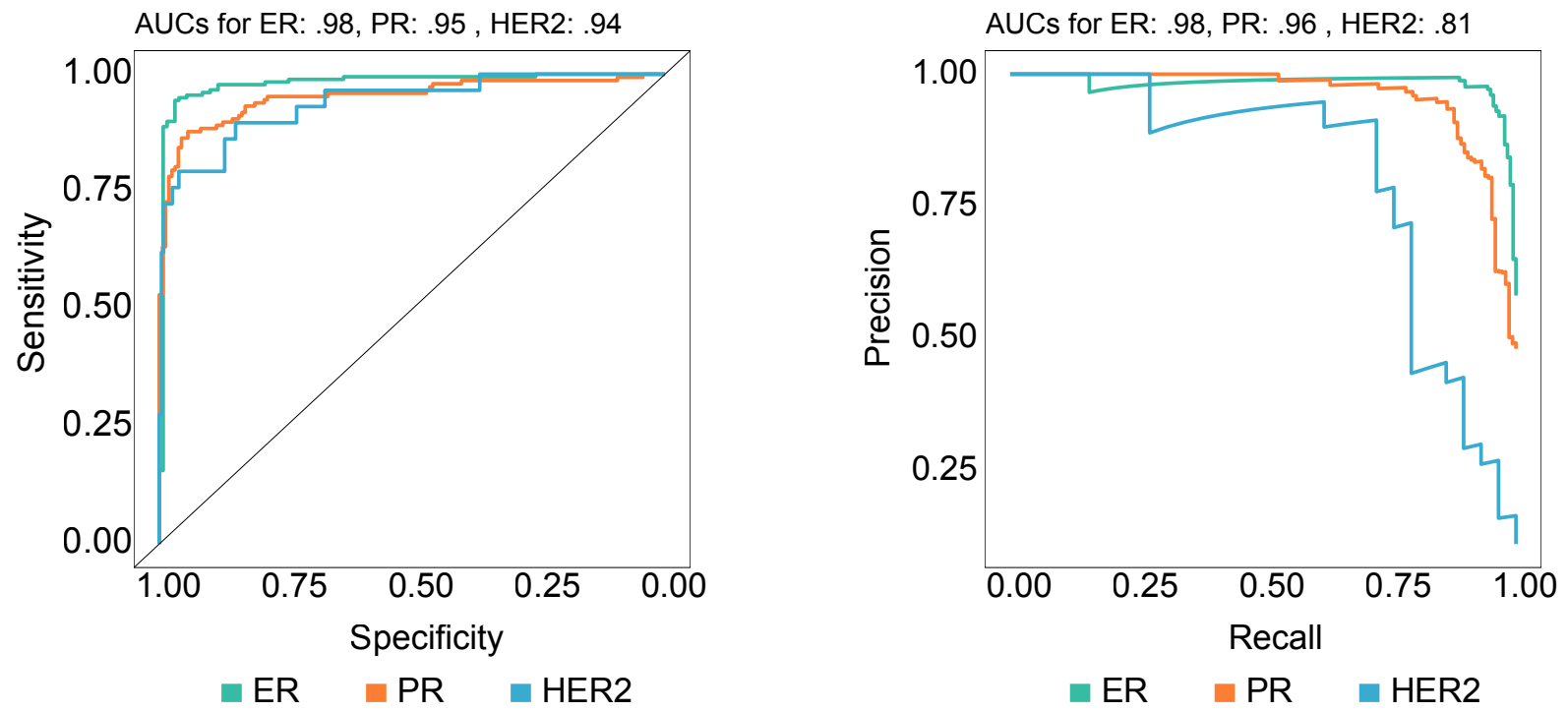

C

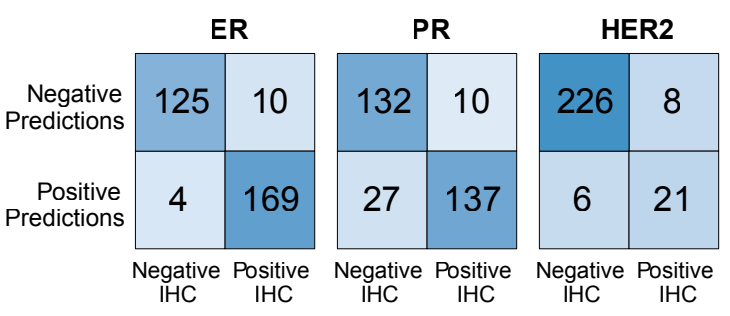




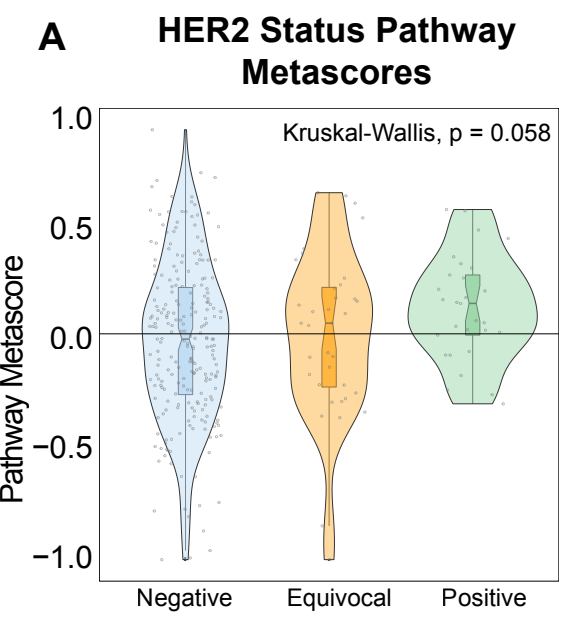

HER2 Status (Abstracted Only)
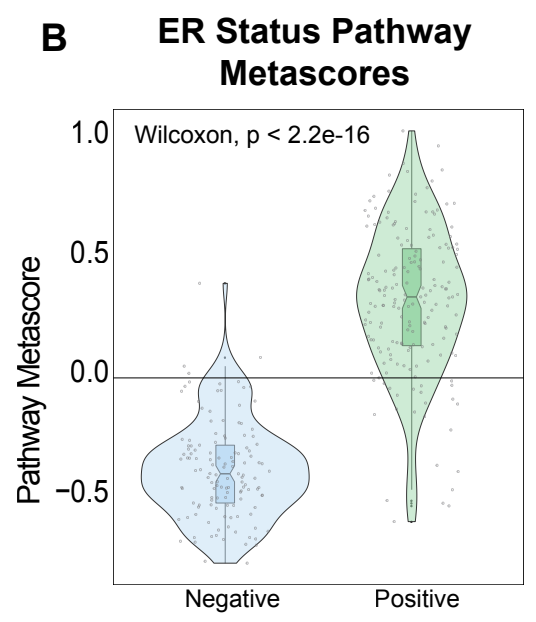

ER Status (Abstracted Only)
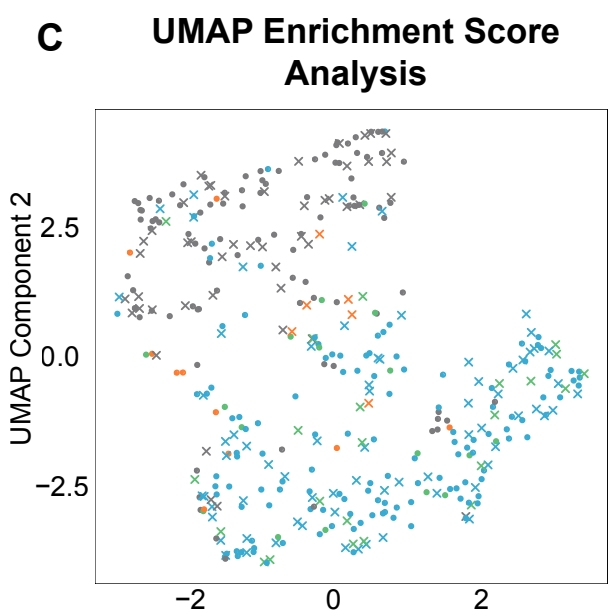

UMAP Component 1

- Abstracted OHR+/HER2- OHR-/HER2+

$\times$ Predicted $\mathrm{HR}+/ \mathrm{HER} 2+$ TNBC
D

HR-/HER2+ Pathway Enrichment

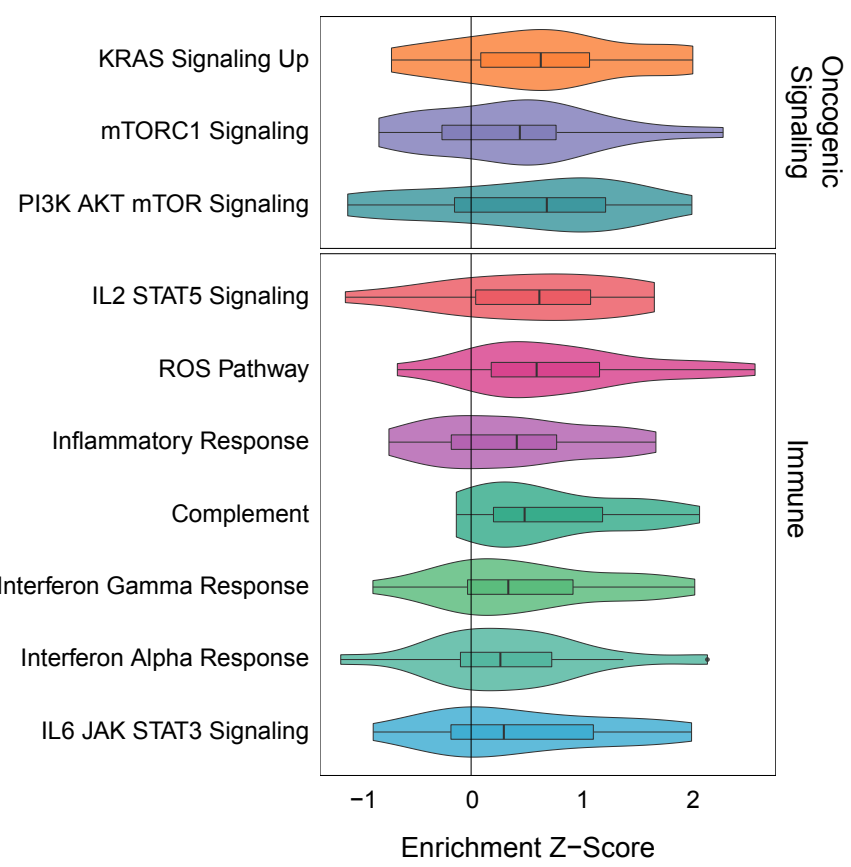

E

\section{TNBC Pathway Enrichment}

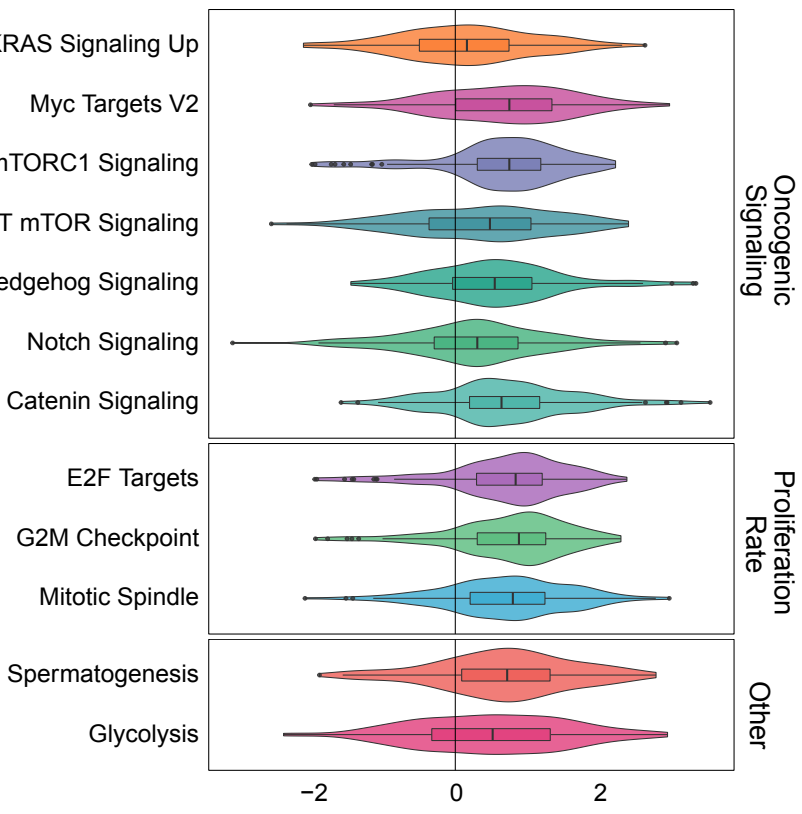

Enrichment Z-Score
PI3K AKT mTOR Signaling

Hedgehog Signaling

Notch Signaling

Wnt Beta Catenin Signaling 
medRxiv preprint doi: https://doi.org/10.1101/2020.08.07.20168401; this version posted August 11, 2020. The copyright holder for this preprint (which was not certified by peer review) is the author/funder, who has granted medRxiv a license to display the preprint in perpetuity.

It is made available under a CC-BY-NC-ND 4.0 International license .

Table 1. Patient demographics and clinical characteristics of the clinical abstraction and Tempus molecular sequenced cohorts at initial diagnosis

\begin{tabular}{|c|c|c|c|}
\hline & & $\begin{array}{l}\text { Clinical Abstraction } \\
\text { Cohort }(\mathrm{N}=4,000)\end{array}$ & $\begin{array}{l}\text { Molecular Sequenced } \\
\text { Cohort }(\mathrm{N}=400)\end{array}$ \\
\hline \multirow[t]{2}{*}{ Sex, n (\%) } & Female & $3,970(99.3 \%)$ & $396(99.0 \%)$ \\
\hline & Male & $30(0.7 \%)$ & $4(1.0 \%)$ \\
\hline \multirow[t]{5}{*}{ Race, n (\%)* } & White & $3,332(83.3 \%)$ & $185(77.1 \%)$ \\
\hline & Black/AA & $523(13.1 \%)$ & $35(14.6 \%)$ \\
\hline & Asian or $\mathrm{PI}$ & $145(3.6 \%)$ & $13(5.4 \%)$ \\
\hline & Other & 0 & $7(2.9 \%)$ \\
\hline & Unknown & 0 & 160 \\
\hline Median age (IQR) & & $61(51.8-70.2)$ & $55.8(45.2-66.4)$ \\
\hline \multirow[t]{6}{*}{ Stage, $\mathrm{n}(\%)^{*}$} & 0 & $42(1.1 \%)$ & 0 \\
\hline & 1 & $1,986(49.6 \%)$ & $30(13.9 \%)$ \\
\hline & II & $1,333(33.3 \%)$ & $83(38.4 \%)$ \\
\hline & III & $420(10.5 \%)$ & $46(21.3 \%)$ \\
\hline & IV & $219(5.5 \%)$ & $57(26.4 \%)$ \\
\hline & Unknown & 0 & 184 \\
\hline \multirow[t]{16}{*}{ Histological subtype, n (\%)* } & Invasive ductal & $3,095(77.4 \%)$ & $267(75.0 \%)$ \\
\hline & Invasive lobular & $345(8.6 \%)$ & $23(6.5 \%)$ \\
\hline & Invasive carcinoma NOS & $214(5.4 \%)$ & $20(5.6 \%)$ \\
\hline & Invasive ductal/lobular & $167(4.2 \%)$ & $20(5.6 \%)$ \\
\hline & Mucinous (colloid) & $61(1.5 \%)$ & 0 \\
\hline & Ductal in situ & $45(1.1 \%)$ & $4(1.1 \%)$ \\
\hline & Tubular & $31(0.8 \%)$ & $1(0.3 \%)$ \\
\hline & Papillary & $15(0.4 \%)$ & $1(0.3 \%)$ \\
\hline & Inflammatory & $8(0.2 \%)$ & $3(0.8 \%)$ \\
\hline & Metaplastic & $6(0.1 \%)$ & $12(3.4 \%)$ \\
\hline & Other & $6(0.1 \%)$ & $3(0.8 \%)$ \\
\hline & Medullary & $4(0.1 \%)$ & 0 \\
\hline & Lobular in situ & $1(0.03 \%)$ & $1(0.3 \%)$ \\
\hline & Unmapped malignancy & $1(0.03 \%)$ & 0 \\
\hline & Phyllodes & $1(0.03 \%)$ & $1(0.3 \%)$ \\
\hline & Unknown & 0 & 44 \\
\hline \multirow[t]{4}{*}{ Menopausal status, n (\%)* } & Postmenopausal & $1,867(86.8 \%)$ & $67(91.8 \%)$ \\
\hline & Premenopausal & $285(13.2 \%)$ & $6(8.2 \%)$ \\
\hline & Unknown & 1,818 & 313 \\
\hline & Not applicable $^{\dagger}$ & 30 & 4 \\
\hline
\end{tabular}

IQR, interquartile range; AA, African American; PI, Pacific Islander; NOS, not otherwise specified *Patients with unknown, unreported, or not applicable characteristic/demographic data were not included in population percentage comparisons.

${ }^{\dagger}$ Represents male patients in the cohort. 